\title{
An Optical Approach for the Determination of Droplet Volumes in Nanodispensing
}

\author{
Kerstin Thurow, ${ }^{1}$ Thomas Krüger, ${ }^{1}$ and Norbert Stoll ${ }^{2}$ \\ ${ }^{1}$ Center for Life Science Automation, F.-Barnewitz-Street 8, 18119 Rostock, Germany \\ ${ }^{2}$ Institute of Automation, University of Rostock, R.-Wagner-Street 31, 18119 Rostock, Germany \\ Correspondence should be addressed to Kerstin Thurow, kerstin.thurow@celisca.de
}

Received 5 February 2009; Accepted 10 February 2009

Recommended by Peter Stockwell

This paper looks at the use of drop-on-demand technology in automated life science laboratories. One significant feature of the droplets generated is the enormously high uniformity under constant conditions. However, there is also a relatively strong dependency on environmental conditions. Many different kinds of liquids are used in laboratory applications, some with unknown properties in advance. In light of this and the special way in which the droplets are created, there is one major question which needs answering in relation to the use of this technology in the area of laboratory automation: What is the volume of the drops generated? This paper will present a solution which can be applied in practice. Besides the technical implementation demonstrated, further alternatives will also be presented. Ultimately, dosing systems with greater autonomy should result from the optimizations demonstrated in this paper.

Copyright (C) 2009 Kerstin Thurow et al. This is an open access article distributed under the Creative Commons Attribution License, which permits unrestricted use, distribution, and reproduction in any medium, provided the original work is properly cited.

\section{Introduction}

Inkjet dispensing technologies have an increasing number of applications in chemical and biotechnological applications with a major focus on drug discovery [1-5]. With the ability to generate any number of drops with nanodspensing devices, larger quantities can be generated very precisely. However, drop volume is not a constant as it depends on the properties of the liquid and the dosing parameters. In the case of this technology and design, an individual drop has a volume of between $50-500 \mathrm{pl}$. There are procedures for measuring individual drops and methods for measuring dosed quantities in nanoliters. However, these methods may be based on very complex measurement technology and/or not allow for real-time measurement of the volume.

Thus, the goal of this paper is to find a procedure for the real-time measurement of the volume of a drop. Finding a balance between the effort and benefit involved in integrating this into the existing equipment is to the fore in this, along with practical use in laboratory automation.

Due to the properties of liquids, such as density, viscosity, and surface tension, different levels of activation energy must be applied to eject the drops. At the same time, the duration of the pressure pulse has an important role to play. Liquids will only be pushed out of the nozzle if the values are aligned. However, this is not all that is involved in the calibration process. The drops being formed must meet certain standards in order to ensure high-quality dosing.

\section{Classical Procedures for Volume Measurements}

Liquid dispensers based on piezoelectric technology can currently dispense the smallest volumes. The amount to be dispensed is quantizised in the specific generation of individual drops with almost identical properties. However, such high reproducibility is only possible under constant conditions. The drop volume depends on the dosing parameters, the liquid, and the nozzle used. Therefore, the size of the drops generated is unknown for each new configuration. However, dosing of large volumes relies on a defined number of drops with a known volume being dispensed. Thus, the dosing quantity is the product of the drop volume and number of drops. Even given larger numbers of drops, 
measuring the volume is technically challenging due to the small quantities, in the nanoliter and microliter ranges.

\subsection{Analytical Measurement Procedures. There are several} methods applicable for the determination of the volume of a droplet. Usually they are offline methods which require the dosing of a defined number of drops prior to the analysis. Conclusions on the volume of an individual drop are arrived at by using the knowledge of the number of drops, specific additional information depending on the procedure, and a likewise procedure-specific measured physical property.

Volume determination using weight is the procedure that requires the least effort. The volume can be calculated using the density of the liquid. A high-precision weighing scale is required for this method. Disadvantages of this method include the fact that the density of the substance must be known, and that there is a risk of evaporation. In respect of increasing the level of automation, the technical realization is in the realm of the possible. However, due to the limitations, it does not really represent a viable alternative but could be used as a reference procedure for the visual procedure presented in this paper [6].

Another method for the determination of the volume is the use of chromatographic procedures. In this procedure, a substance with a specified volume is used as a reference, and a defined number of drops are added to it. This mixture of substances can be analyzed using liquid or gas chromatography. The volume is calculated using the measured concentration and the known quantity of the reference substance used. The reference substance must also be dosed with great precision. Furthermore, the analysis is based on very complex measurement technology $[7,8]$.

Fluorescence measurement is also based on a concentration measurement. A substance with known fluorescence properties is added to the wells of a microtiter plate in a test series with an increasing number of drops. The known ratio of the measured radiation of the respective fluorescent substance is used to determine the volume. Besides the limitations mentioned, complex measurement technology is also needed for this [9].

\subsection{Real-Time Measurement Procedures from Particle Mea-} surement Technology. Procedures based on optical instruments for the very precise measurement of generated drops already exist in the area of particle measurement technology. The measurement technology facilitates real-time recording; but very complex hardware is required.

The spatial filter technology method, now established in particle and flow measurement technology, involves the use of cameras with CMOS sensors which, unlike CCD sensors, allow for individual areas, rows, and even pixels to be read out. The underlying operational principle is that of lattice diffraction. The lattice is replaced by a line scan or matrix camera. The lattice constant is then derived from the size and distance of the individual pixels. If an object moves along a row, a characteristic signal emerges after the summation due to the alternating weighting (positive, negative) of the individual pixel signal. The signal is evaluated on the basis of a frequency analysis. When knowledge of the properties of the optical system, pixels, readout characteristics, and evaluation electronics are combined, the speed of the particle can be calculated [10]. The particle size can be determined if the speed is known. As the readout cycle has to be significantly faster than the movement of the measurement object, sampling rates in the $\mathrm{MHz}$ range are required. These can be achieved using CMOS sensors and DSPs for signal processing $[11,12]$.

The laser measurement technology is a high-resolution procedure from particle measurement technology based on the illumination of a drop with a laser. On penetrating the liquid, refraction occurs in accordance with the properties of the liquid. These refractions are captured by a camera. Small light spots can be seen in the focused image. By using a defocused camera, there is an overlapping of this spots. The characteristic of the overlap of the two points results from the refraction index of the liquid. By counting the fringes in the defocused picture, the drop diameter can be determined.

The level of precision in determining the drop diameter is up to $1 \mu \mathrm{m}$. However, the drops have to have a spherical shape. The procedure is only suitable for measuring size. There are other procedures which rely on this measurement principle. They are based on more extensive technology but also deliver more precise and enhanced results. Another advantage of this procedure is that it can be applied without there being any link to the control of the dosing head. This means that no information is required about the dosing or stroboscope parameters. By dosing using this device, volume can be measured. However, no information regarding other criteria in the dosing procedure can be obtained by using this procedure.

The procedures based on particle measurement technology are suitable for both very precise real-time measurement of the drop volume and also automated implementation. However, very complex measurement technology is needed for them, and the expense required to integrate them into commercial equipment may not be justifiable in certain circumstances.

\section{Analysis of the Dispensing of a Drop}

Capturing the dosing procedure was the basis for all further steps. This aim can be achieved using various technologies. These range from different models of simple light barriers and cameras to laser-guided measuring procedures. With regard to all measurement methods, practical applicability was of particular interest and led to a compromise between the resolution of the procedure and the integration complexity. Furthermore, the procedure had to be digitalized for automated evaluation.

The drops generated had an average volume of approximately $250 \mathrm{pl}(\varnothing 78 \mu \mathrm{m})$ and flew with a velocity of about $2 \mathrm{~m} / \mathrm{s}$.

3.1. Using Measurement Technology to Capture the Drop Flight. Visualization is usually implemented in most appliances with the aid of a stroboscope camera system. This is 


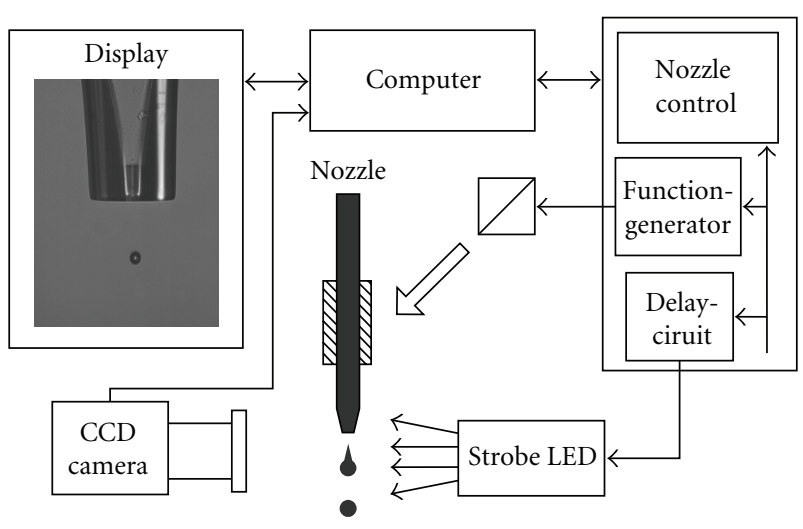

FIGURE 1: Overview of the nozzle's visualisation and control system.

already of great benefit in the manual evaluation of drop formation. The camera image can be imported into the dosing software using a frame grabber or USB camera, for example. Some of the dosing robot's control applications already applied simple image processing algorithms to monitor drop formation.

3.1.1. Visualization Using a Stroboscope and Camera. The nanodoser used in the tests here, S-5 from Scion, has a stroboscope camera system and thus the technical requirements for monitoring drop formation. The existing hardware is made up of a camera with an enlarging lens and an LED stroboscope. The stroboscope was needed as the drops move too quickly to get an individual image of them with a conventional camera. The synchronous triggering of the stroboscope with the activation from the piezoelectric crystal provided an image of overlapping drops in continuous operation. In addition to this, it was possible to change the delay between the rising flanks of the piezoelectric signal and the activation of the stroboscope. By doing so, the drops could be visualized at any point in time after being dispensed [13-15]. (See Figure 1.)

In order to get an idea of the temporal progress of the drop ejection, the stroboscope flash delay was continuously increased from zero upwards. By doing so, still images of the continuous process could be generated at different points in time.

3.1.2. Optical Component Parameters. The lens with aperture, intermediate rings, and camera sensor are the most important parts of the system. It was possible to calculate selected statistical values on the basis of this setup [16, 17].

$\beta$ Reproduction Scale. This depends in particular on the lens and its focal length and position in the optical system;

$$
\beta=\frac{l}{f}
$$

where $l$ : extension length and $f$ : focal length.
TABLE 1: Technical data from the camera's CCD sensor.

\begin{tabular}{lccc}
\hline & $\begin{array}{c}\text { Number of pixels } \\
\left(P_{a}\right)\end{array}$ & $\begin{array}{c}\text { Pixel size } \\
\left(P_{g}\right)\end{array}$ & $\begin{array}{c}\text { Sensor size } \\
\left(P_{a} * P_{g}\right)\end{array}$ \\
\hline Horizontal & 768 & $8.4 \mu \mathrm{m}$ & $6451 \mu \mathrm{m}$ \\
Vertical & 494 & $9.8 \mu \mathrm{m}$ & $4841 \mu \mathrm{m}$ \\
\hline
\end{tabular}

For the lens used,

$$
\beta=\frac{l}{f}=\frac{l_{\text {lens }}+l_{\text {tube }}}{f_{\text {lens }}}=\frac{35 \mathrm{~mm}+30 \mathrm{~mm}}{12 \mathrm{~mm}}=\frac{65}{12}=\underline{\underline{5.42}} .
$$

Pixel $\leftrightarrow$ Micrometer Conversion Factor. The reproductionscale data from the individual CCD sensor and the resolution of the camera can be used to determine this factor. (See Table 1.)

Thus, 768 pixels are the maximum vertical image size possible of $B_{\text {vertical }}=6451 \mu \mathrm{m}$. The object size can be calculated using the reproduction scale and the $\beta=B / G$ formula:

$$
G=\frac{B}{\beta}=\frac{B_{\text {horizontal }}}{\beta}=\frac{6451 \mu \mathrm{m}}{5.42}=\underline{\underline{1191 \mu \mathrm{m}}} .
$$

For the individual pixels, this resulted in

$$
\frac{G}{P_{a}}=\frac{1191 \mu \mathrm{m}}{768 \text { pixels }}=\underline{\underline{1.55 \mu \mathrm{m} / \text { pixels. }}}
$$

Analogous to this, the width of the image was vertically calculated using

$$
G=\frac{B}{\beta}=\frac{B_{\text {vertical }}}{\beta}=\frac{4841 \mu \mathrm{m}}{5.42}=\underline{\underline{894 \mu \mathrm{m}}} .
$$

The CCD sensor consisted of 576 rows. Thus, for the conversion in the vertical direction, we get

$$
\frac{G}{P_{a}}=\frac{894 \mu \mathrm{m}}{576 \text { pixels }}=\underline{\underline{1.55 \mu \mathrm{m} / \text { pixels. }}}
$$

Depth of Field of the System. This parameter describes the space between two planes which are vertical to the optical axis. Focusing is optimal in the region between the planes. The distance between these planes depends on the optical components mentioned and the f-number. The f-number is defined and standardized in accordance with DIN 4522 and describes the ratio between the focal length and the diameter of the lens' entrance pupil:

$$
\kappa=\frac{f}{D},
$$

where $f$ : focal length and $D$ : diameter of the entrance pupil.

Further requirements, also calculable from the system data, are the dispersion circle diameter and the hyperfocal distance. The dispersion circle diameter is determined using a generally accepted approximation formula:

$$
Z=\frac{d}{1730} \quad \text { from [112], }
$$

where $d$ : diagonal of the camera's CCD sensor. 
TABLe 2: Depths of field for particular $f$-numbers.

\begin{tabular}{lccc}
\hline & $\kappa=2$ & $\kappa=4$ & $\kappa=8$ \\
\hline Near point & $17.555 \mu \mathrm{m}$ & $17.549 \mu \mathrm{m}$ & $17.536 \mu \mathrm{m}$ \\
Far point & $17.568 \mu \mathrm{m}$ & $17.574 \mu \mathrm{m}$ & $17.587 \mu \mathrm{m}$ \\
Depth of field & $12 \mu \mathrm{m}$ & $25 \mu \mathrm{m}$ & $51 \mu \mathrm{m}$ \\
\hline
\end{tabular}

For the apparatus used here, we get

$$
\begin{aligned}
Z & =\frac{d}{1730}=\frac{\sqrt{B_{\text {horizontal }}^{2}+B_{\text {vertical }}^{2}}}{1730} \\
& =\frac{\sqrt{(6451 \mu \mathrm{m})^{2}+(4841 \mu \mathrm{m})^{2}}}{1730}=4.7 \mu \mathrm{m} .
\end{aligned}
$$

With a dispersion circle diameter $Z$, we can calculate the hyperfocal distance $d_{h}$ :

$$
d_{h}=\frac{f^{2}}{Z \cdot \kappa},
$$

where $f$ : focal length of the lens.

The near and far points, the limits of the depth of field, were calculated using $d_{n}=g \cdot d_{h} /\left(d_{h}+(g-f)\right)$ for the near point, $d_{f}=g \cdot d_{h} /\left(d_{h}-(g-f)\right)$ for the far point.

The difference between the far and near points is the region of optimal focus. The values calculated for selected $\mathrm{f}$ numbers for the lens used are shown in Table 2 . The favoured aperture setting for the measurements is $\kappa=4$.

Information on the depth of field increases in significance when combined with the determination of the focusing. With these two statistical values, the three-dimensional trajectory of the drop can be determined.

3.2. Algorithm for Extracting the Image Data. With the aid of image processing algorithms, the drop data can be extracted from the image generated by the visualisation system described. In order to be in a position to capture the drops even given insufficient focusing or unfavourable lighting conditions, the algorithms must be designed to be robust. This procedure is described in the following sections. Optimal focusing on the drop is a precondition to actually determining the drop parameters [18].

Four steps are necessary for extracting the image data.

3.2.1. Isolation of the Region of Interest. This step establishes which region of the image should be inspected for a drop. This is statically determined. It is possible to do this as the positioning of the nozzle in front of the camera is defined and carried out in advance. Another reason for the limitation is that the drop is usually dispensed vertically. Reducing the image region to be analyzed also reduces the computational effort required. For these reasons, the region of interest was limited to the middle third of the lower half of the image.

3.2.2. Creation of the Binary Image. A binary image is the basis for further algorithms for determining the properties of the drop. The black and white images in this case consist

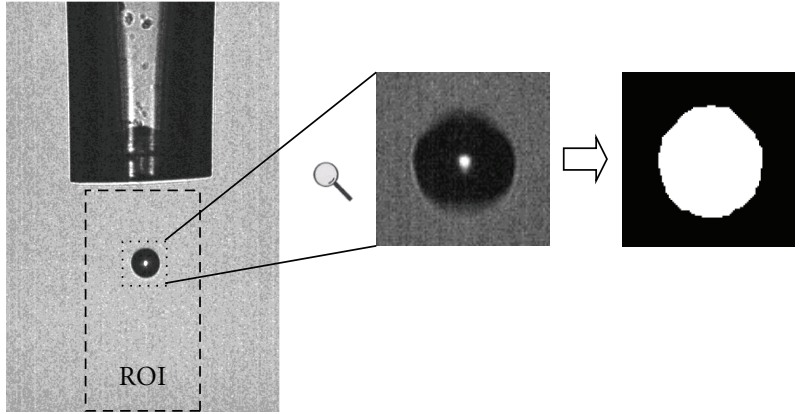

FIGURE 2: Generation of the binary image from the region of interest.

of pixel values of 0 for black and 255 for white. A threshold is needed for the division. It is calculated dynamically from the mean value of the grey-scale values in the image. The fact that the drops displayed are always darker than the background is used for this. The actual threshold is calculated by multiplying the mean value by the correction factor 0.9 for a robust measurement. (See Figure 2.)

Following the conversion, the image consists of a white background (TRUE or 255) and the drop (FALSE or 0). However, this setup is disadvantageous for further calculations as the drop is the object of interest. Therefore, a negative is generated after the conversion.

\subsubsection{Preparation of the Drop Contour}

Morphological Opening. Due to the type of visualization and associated blurred edge, the binary image was morphologically opened with a circle. By doing so, the edges of the remaining contour were smoothed, and individual interfering pixels which had remained were eliminated [19, 20].

Deleting Objects at the Edge. This step is necessary to remove unwanted contours at the edge of the image, a result of unfavourable light conditions, and to avoid measurement errors caused by drops that have only been partially mapped. The first point is secondary in importance given good lighting conditions. However, the second aspect is significant once the drop image is located at the edge of the image during the measurement. If the drop is measured despite touching the edge, this will lead to considerable measurement errors if only a fraction of the true size of the image is within the region captured.

Filtering out Unwanted Contours. Individual contours and those remaining from the preceding steps which do not represent drops must be filtered out. These contours can be caused by luminance noise, dust on the lens, and the like. The principle approach in this is the systematic eradication of all remaining contours. The area of the contour is the criterion for elimination. Connected areas bigger than 300 pixels should be kept. The threshold for an area is a matter 


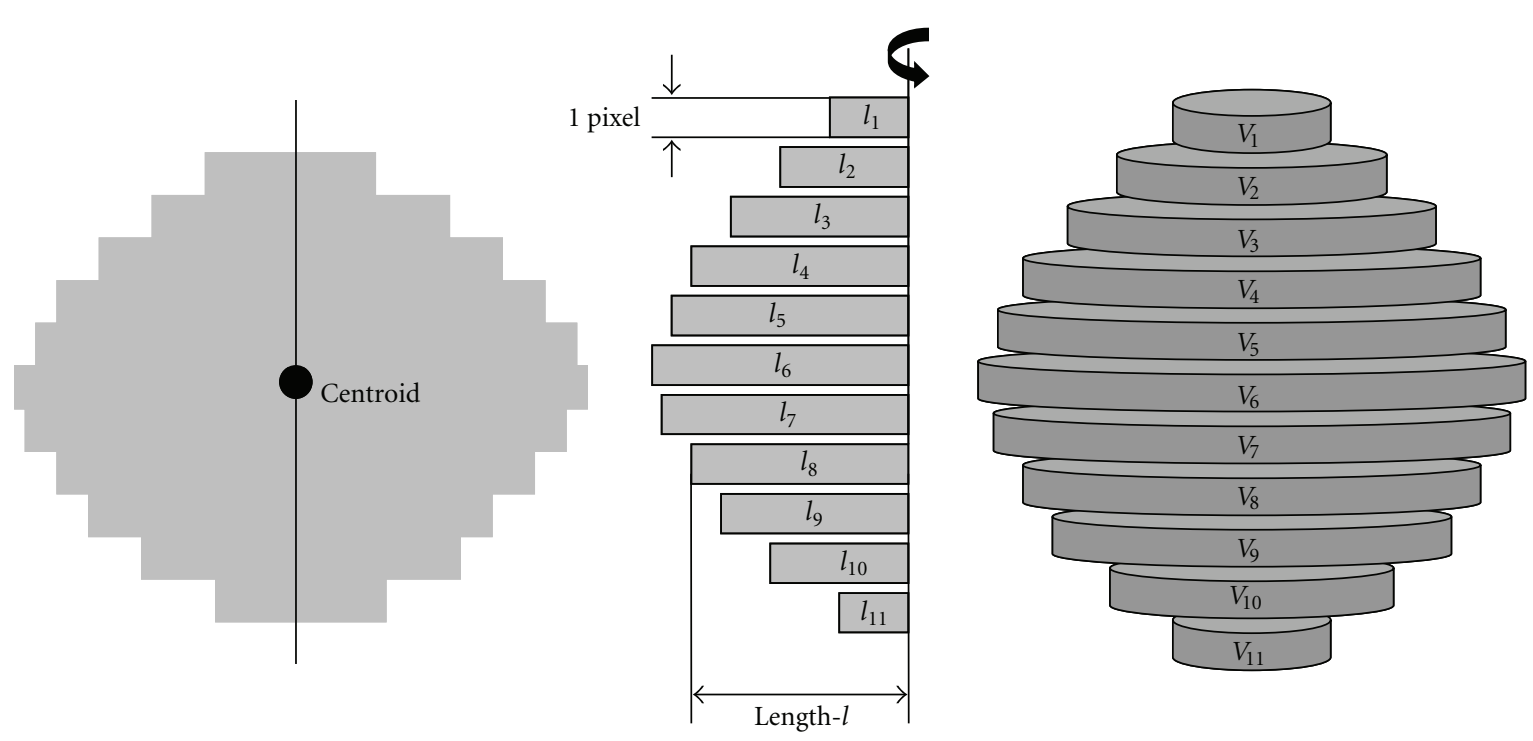

Figure 3: Principle by which the drop volume was calculated.

of experience. This procedure removes almost all interfering contours but still permits satellite drop detection.

Filling of Holes. Usually, light focused in the centre of the drop is brighter than at the edge. Therefore, the drop contour has a hole in the middle following inversion. Filling is necessary for further calculations and was carried out using an algorithm in the image processing library. This was systematically applied to the remaining contours in the image. The result of this operation was the basis for all further procedures used to gain information on the drop.

\subsubsection{Projecting the Drop Detection into the Camera Image.} The drop detected was superimposed onto the original image in order to get an impression of the actual capturing of the drop image. In addition to this, the edge line of the drop contour from the final binary image was laid in a visibly light tone over the captured frame.

This illustration of the results of the image processing is particularly important in development. The developer or user can monitor the extent to which the drop detection corresponds with the reality. Although this is a subjective finding, individual parameters, such as the conversion threshold, can be aligned in the binary image.

3.3. Volume Calculation from the Drop Image. There are several ways in which a three-dimensional body can be calculated from a two-dimensional drop contour. The radius or the 3 axes of the contour can be used as a basis if a spherical shape or ellipsoid is assumed. Another method, and the one used here, is the procedure shown in Figure 3. However, both calculations rely on the assumption that the drop is rotationsymmetric in the direction of flight.

The volume of a slice is

$$
V_{n}=\pi r_{n}^{2} \cdot d,
$$

where $d$ : unit thickness of 1 pixel and $r$ : radius of the slice/width of the strip.

The volume of the rotational body of half of the drop is

$$
V_{\text {drop }}=\sum_{n=1}^{L} V_{n}=\sum_{n=1}^{L} \pi r_{n}^{2} \cdot d,
$$

where $d$; unit thickness of 1 pixel, $L$ : length of the drop, and $r$ : radius of the slice/width of the strip.

The contour is cut into strips in order to calculate the volume of the rotational body of the respective half. These strips run vertically to the axis of symmetry and are each one pixel in thickness. Slices of equal thickness are then calculated from these strips. This process is carried out for each strip of the respective half in sequence. Following this, the volumes of the individual slices are added together. The result of this sum corresponds to the volume of the drop. This process is carried out in the same way for the other half of the drop. The mean value from both volumes is taken to be the final volume.

Another approximation can be made by calculating a sphere or ellipsoid. If we assume a sphere, the width of the contour is equal to the diameter. Likewise, the width and height of the drop contour provide two of the three axes required. The third axis is the width, assuming rotational symmetry. However, the disadvantage of these methods, as opposed to the division into slices, is that there is always imprecision in respect of the shape deviation. This phenomenon also exists with the strip method but does not play as comparatively great of a role.

The contours, their centroids, and the data of the "bounding rectangle" are needed to calculate the volume. With the aid of the "bounding rectangle," the limits of the contour and the calculation can be determined. The centroid is used to establish the vertical axis of symmetry.

The volume calculated was in units of voxels $\left(\right.$ pixel $\left.^{3}\right)$. For further use, this was converted into picoliters with the aid of 
the optical system calculations, with one voxel corresponding to a cube with an edge length of $1.55 \mu \mathrm{m}$.

3.4. Influences on the Determination of the Droplet Volume. Different conditions have an impact on the determination of the precise droplet volume.

3.4.1. Influence of Different Drop Shapes. There is a certain tolerance with respect to different drop shapes when calculating drop volumes according to the integration procedure. However, there can be large fluctuations in the volumes calculated if there are extreme contortions in the drop shape. It may not be appropriate to assume rotationally symmetric drops in some cases. The drop shape can fluctuate strongly at the beginning of the flight phase in particular due to the forces which arise during release, and symmetry is not given at that time.

One way of circumventing this interference effect is to only capture the drop after it has gone a certain distance. The drop shape becomes increasingly stable and strives for a perfectly spherical shape. The disadvantage of this is that the drop has to travel a distance of approximately $2 \mathrm{~mm}$. The recording of the drop at another position or at another time requires additional effort as the dosing head has to be put up high, and the delay in the LED stroboscope has to be adjusted.

A second method involves averaging the volume values measured across the drop's entire flight phase. When applying this method, the nozzle position in front of the camera does not need to be changed. This procedure was used for the volume measurements carried out here. A third alternative, carrying out the volume measurement at a defined point, is possible with the help of evaluation of the circularity of the drop. For example, this could be the stroboscope delay/flight position at which the drop has the greatest circularity in the trackable trajectory. Both the strip method and the calculation of the spherical volume can be used in this.

3.4.2. Enlarged Appearance of the Drops. Using a frequency of $500 \mathrm{~Hz}$ and a stroboscope light duration of 5 microseconds, a volume 1.17 times greater than the weighing was arrived at with the measurements from the procedure based on image processing. This behavior is a consequence of the visualization principle. The camera used did not have a fast shutter or triggering. Therefore, the duration of the stroboscope flash was crucial for the size of the appearance of the drops.

3.4.3. Frequency Dependency of the Correction Factor. When the measurements were carried out at different frequencies, the systematic error increased as can be seen in Figure 4 .

The frame rate of the camera used, a Sony XC-ES 50, is 30 images per second. The camera was not triggered via the stroboscope signal. The shortest possible shutter speed was 100 microseconds, far longer than the light duration of the LED stroboscope. The number of drops overlapping in the picture depends on the dosing frequency [21].

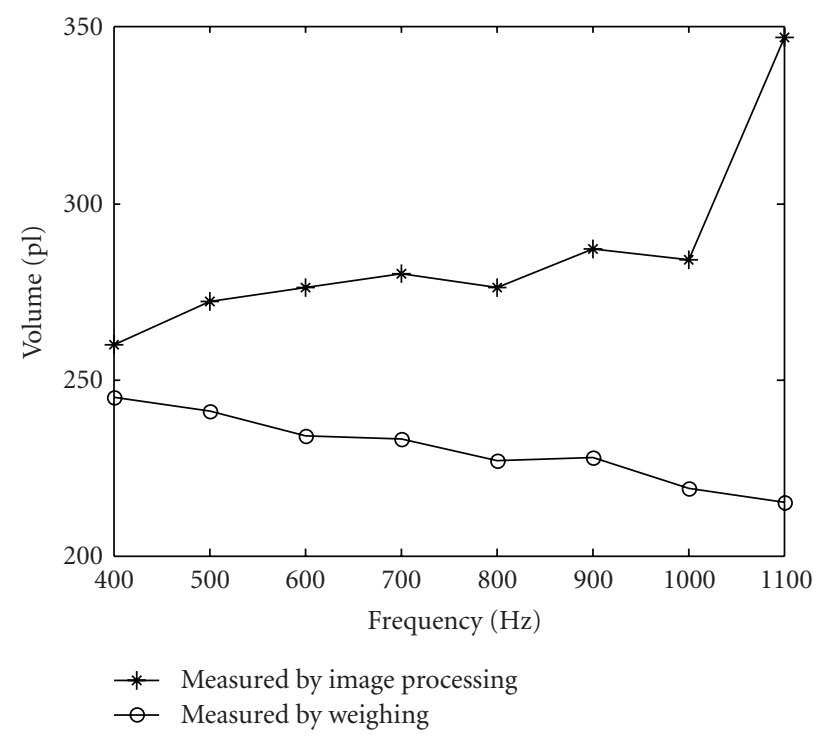

FIGURE 4: Frequency dependence of the dispensed volume.

TABLE 3: Number of overlapping drops in the image depending on the drop rate.

Drop rate $400500600 \quad 8001000 \quad 15002000300040005000$ $(\mathrm{Hz})$

$\begin{array}{lllllllllll}\text { Overlapping } & 13 & 16 & 20 & 26 & 33 & 50 & 66 & 100 & 133 & 166\end{array}$ drops

As the number of overlapping drops in an image increases, the effect described below has a greater influence. The distance between the drops in the droplet jet generated can vary periodically. As the frequency increases, the drops appear much larger than they would otherwise. However, this phenomenon does not always appear and is not predictable. It was not possible to detect it with the methods shown here. (See Table 3.)

The measurements taken show that the systematic error increases sharply in frequencies above $800 \mathrm{~Hz}$ because of this effect. Conditional on the fact that the fluctuation in the drop distance cannot be detected with the procedures used here, it was not advisable to measure the volume with the procedures shown at higher frequencies.

3.4.4. Uncertainty in Edge Detection. Besides the uncertainties in the recording technology, the image processing procedure can also influence the measurement error. The edge detection has a crucial weighting. The slices of which the drop volume is comprised were calculated on the basis of this edge data. Using the example of the calculation of a sphere, Table 4 illustrates the degree to which the incorrect detection of the radius can affect the entire volume.

3.4.5. Illumination from the LED Stroboscope. The environmental light is another influencing factor. It is hard to take all fluctuations into account. In order to counter the effects of external light influences, a box is recommended. The entire 
TABle 4: Estimate of the difference in volume if the edge is incorrectly detected.

\begin{tabular}{lccc}
\hline Drop volume & $\begin{array}{c}\text { Diameter } \\
\text { (rounded) }\end{array}$ & $\begin{array}{c}\text { Difference in } \\
\text { volume with } \\
a-1 \text { diameter }\end{array}$ & $\begin{array}{c}\text { Difference in } \\
\text { volume with } \\
a+1 \text { diameter }\end{array}$ \\
\hline $100 \mathrm{pl}$ & 37 pixels & $7.9 \%$ & $8.3 \%$ \\
$150 \mathrm{pl}$ & 43 pixels & $6.8 \%$ & $7.1 \%$ \\
$200 \mathrm{pl}$ & 47 pixels & $6.2 \%$ & $6.5 \%$ \\
$250 \mathrm{pl}$ & $\mathbf{5 0}$ pixels & $\mathbf{5 . 9} \%$ & $\mathbf{6 . 1} \%$ \\
$\mathbf{3 0 0} \mathbf{~ p l}$ & $\mathbf{5 4}$ pixels & $\mathbf{5 . 5} \%$ & $\mathbf{5 . 7} \%$ \\
$\mathbf{3 5 0} \mathrm{pl}$ & $\mathbf{5 6}$ pixels & $\mathbf{5 . 3} \%$ & $\mathbf{5 . 5} \%$ \\
$400 \mathrm{pl}$ & 59 pixels & $5.0 \%$ & $5.2 \%$ \\
$450 \mathrm{pl}$ & 61 pixels & $4.8 \%$ & $5.0 \%$ \\
$500 \mathrm{pl}$ & 64 pixels & $4.6 \%$ & $4.8 \%$ \\
\hline
\end{tabular}

unit was covered by a nonlight transparent box for the tests carried out here.

The light needed was generated by the visualization system itself. The light duration and frequency of the LED stroboscope were thus primarily responsible for the brightness of the image.

As the brightness and thereby changed contrast ratios in the image influence the drop detection procedure, it is recommended that measurements should be taken with the most constant illumination possible. The ratio of the greyscale values in the background to those of the drop is reduced at both high and also low intensities of the light source. The detection procedure fails in these limit ranges despite a certain robustness in the edge detection. However, the brightness must be standardized to ensure measurements with the lowest influence of error possible. Therefore, it is recommended that constant aperture, camera settings, and constant stroboscope parameters to be used when taking the measurements.

\section{Verification Measurements}

In order to check the findings and ensure their general validity, a number of test series were performed. Due to the dependency of the systematic error on the dosing frequency, stroboscope frequency, and light duration, these values were not varied. However, this did not limit the regular operation.

Gravimetric recording was used as a reference for the measurement of the volume with the method presented. In order to cover a number of examples in relation to the range of applications, dosing was implemented using the following substances.

4.1. Gravimetric Measurement as a Reference. The uniformity of the drops in the jet is always given when using aligned dosing parameters. This statement can be made because of the method of drop generation and the measurement findings of the processed camera image. In order to minimize the error caused by evaporation, dosing was carried out into an Eppendorf tube already filled with the respective solution.
TABLE 5: Composition of the parameters of the stroboscope camera system for the standardized measurement.

\begin{tabular}{lll}
\hline Camera & $\begin{array}{l}\text { Camera frame rate } \\
\text { Minimal shutter speed } \\
\begin{array}{l}\text { Triggering and } \\
\text { synchronization }\end{array}\end{array}$ & $\begin{array}{l}30 \text { images/s (full } \\
\text { frame) } \\
100 \mu \text { s (not used) }\end{array}$ \\
$\begin{array}{l}\text { Control pulse of } \\
\text { the piezoelectric } \\
\text { element }\end{array}$ & Dosing frequency \\
Stroboscope & $\begin{array}{l}\text { Stroboscope frequency } \\
\text { Light duration of the LED } \\
\text { stroboscope }\end{array}$ & $500 \mathrm{~Hz}$ \\
Lens & Conversion factor pixel: $\mu \mathrm{m}$ & $1: 1.55$ \\
\hline
\end{tabular}

Table 6: Overview of the liquids used for testing and their properties.

\begin{tabular}{lcc}
\hline Medium & Density & Viscosity \\
\hline Water & $1 \mathrm{~g} / \mathrm{cm}^{3}$ & $1 \mathrm{mPa} \mathrm{s}$ \\
DMSO & $1.10 \mathrm{~g} / \mathrm{cm}^{3}$ & $1.99 \mathrm{mPa} \mathrm{s}$ \\
NMP & $1.03 \mathrm{~g} / \mathrm{cm}^{3}$ & $1.65 \mathrm{mPa} \mathrm{s}$ \\
Acetonitrile & $0.63 \mathrm{~g} / \mathrm{cm}^{3}$ & $0.57 \mathrm{mPa} \mathrm{s}$ \\
Dichloromethane & $1.33 \mathrm{~g} / \mathrm{cm}^{3}$ & $0.58 \mathrm{mPa} \mathrm{s}$ \\
Methanol & $0.76 \mathrm{~g} / \mathrm{cm}^{3}$ & $0.56 \mathrm{mPa} \mathrm{s}$ \\
\hline
\end{tabular}

A dosing height above the target in the range of $0.5 \mathrm{~mm}-$ $1.5 \mathrm{~mm}$ was chosen so that evaporation during the drop flight could be practically ruled out.

Through electronic control, it was possible to ensure that the desired number of pulses was generated. Due to the knowledge acquired at that stage, the assumption could be made when using the aligned parameters that a drop release would take place with each pulse. However, we did not individually prove each one. Due to these facts, it was ensured in principle that the quantity of liquid given would be quantitatively comprised of the product of the drop volume and number of drops.

The test series which followed consisted of 15 individual measurements in each case, using the same nozzle with the same dosing parameters, with Table 7 showing the gravimetrically determined volumes of water given different amounts of drops. In order to determine the systematic error, the volumes measured from the optical and gravimetric methods were proportionalized.

It should be noted that the range limits of the scale were reached with masses smaller than $0.5 \mathrm{mg}$. This factor was taken into consideration in subsequent measurements and, where needed, the number of drops and thus the resulting mass were increased.

4.2. Results of the Different Solvents. Test series with the liquids listed in Table 6, and different nozzles were carried out in the subsequent testing. The nozzles used had diameters of $50 \mu \mathrm{m}$ and $70 \mu \mathrm{m}$. In order to make direct comparisons 
TABLE 7: Gravimetric measurement of different quantities of drops under constant conditions.

\begin{tabular}{|c|c|c|c|c|c|}
\hline Number of drops & 5000 & 7500 & 10000 & 12500 & 15000 \\
\hline $\begin{array}{l}\text { Number of } \\
\text { measurements }\end{array}$ & 15 & 15 & 15 & 15 & 15 \\
\hline $\begin{array}{l}\text { Mean value } \\
{[\mathrm{mg} \text { or } \mu \mathrm{L}]}\end{array}$ & $0.72 \mathrm{mg}$ & $1.12 \mathrm{mg}$ & $1.48 \mathrm{mg}$ & $1.85 \mathrm{mg}$ & $2.24 \mathrm{mg}$ \\
\hline Std. deviation & 0.0372 & 0.0373 & 0.0312 & 0.0398 & 0.0319 \\
\hline$\%$ Std. deviation & $5.2 \%$ & $3.3 \%$ & $2.1 \%$ & $2.2 \%$ & $1.4 \%$ \\
\hline Visual volume & $200 \mathrm{pl}$ & $200 \mathrm{pl}$ & $200 \mathrm{pl}$ & $200 \mathrm{pl}$ & $200 \mathrm{pl}$ \\
\hline $\begin{array}{l}\text { Visual/gravimetric } \\
\text { ratio }\end{array}$ & 1.40 & 1.33 & 1.35 & 1.35 & 1.34 \\
\hline
\end{tabular}

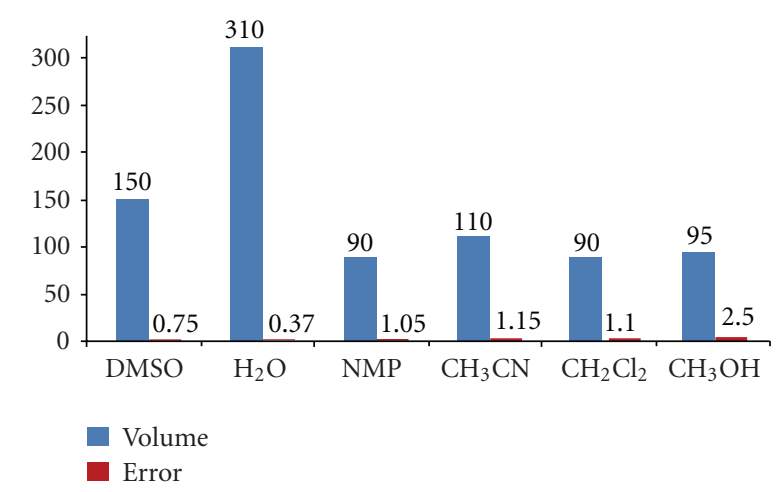

FIGURE 5: Results of optical measurements for different solvents.

between nozzles of the same diameter, two nozzles with a diameter of $50 \mu \mathrm{m}$ were selected.

Both optical and gravimetric measurements were carried out on the water, NMP and DMSO. In the case of the optical measurement, the mean value across the last 32 frames or the associated volume was used in each case. In the weighing, each of these results was made up of 15 individual measurements. In order to ensure additional certainty in the measurement, gravimetric determination was undertaken with different drop numbers, depending on the nozzle and liquid. The number of drops for the first measurement was set at 10000 . The number of drops for the second measurement was aligned with the drop volume determined from the first. If there was a risk of the range limits of the weighing scale being reached in the case of low mass, the drop number was increased to 15000 . If this was not the case, 5000 drops were dosed, thereby accelerating the measurement process and countering evaporation.

The dosing parameters were aligned such that stable drop formation could take place as per the specified quality criteria. $500 \mathrm{~Hz}$ was always used as the dosing frequency. Figure 5 shows the results of the optical measurements for the different solvents.

In the case of the drop volume being measured with the optical method or based on the image processing, different properties of the liquids did not influence the measurement results.

\subsection{Statistics}

Evaluation of the Optical Process. In order to keep the impact of the measurement error low, a mean value was derived for the volume from the last 32 images. The Lilliefor test, a modification of the Kolmogorov-Smirnov test in cases of low numbers of measurement points, was used to test for normal distribution. The null hypothesis was maintained in accordance with this test for a level of significance of 5\%. The mean value and standard deviation were then determined. In the case of stable drop formation, the standard deviation was always between $0.6 \%$ and $1.5 \%$ of the drop volume [22].

Ratio of the Different Volumes Determined. The mean value of the ratio of the two volumes measured in different ways could be determined from the comparative measurements undertaken. Firstly, from the weighing procedure with the aid of the density, and secondly, from the optical process presented. The optical process had a systematic error, explained in Sections 3 to 3.4.5. The weighing scale was used in this case as a reference in order to determine the deviation. The 23 test series in total, carried out under constant lighting conditions and with constant camera settings, showed a mean value of 1.35 with a standard deviation of $0.04(2.93 \%)$.

The deviations in the correction factor of 1.35 in this case and 1.17 from the measurements in Section 1 can be explained by the fact that a new box was constructed between the measurements. The camera settings then had to be adjusted accordingly.

Nevertheless, these values are only valid for a dosing and stroboscope frequency of $500 \mathrm{~Hz}$ and a stroboscope lighting duration of 5 microseconds. For higher dosing frequencies, it is expected and has already been experimentally confirmed in Section 3.4.3 that both the ratio of the optically measured and real volume and its distribution increase. This effect is based on the principle of the visualization of the drops.

4.4. Conclusion on the Overall Measurement Uncertainty. The measurement uncertainty of the stroboscope, the overlapping of several drops on an image, and the movement of the drops effected a measurement uncertainty of approximately $10 \%$. In addition to this, there was uncertainty in relation to the edge detection. This was related to the threshold determination and also to the resolution of the camera's CCD sensors (8-bit). The linear law of the propagation of uncertainty could not be applied as the measurements, and further processing of the measured values was not independent of one another.

In summary, a systematic error arose due to the recording technology and was primarily determined by the parameters: light duration of the LED stroboscope, frequency of the drops, frequency of the stroboscope, and camera frame rate.

In order to keep the error as constant as possible, the measurements here were all carried out with the same dosing frequency $(500 \mathrm{~Hz})$. In the present system, the stroboscope frequency corresponded to the drop rate as these systems were linked. Likewise, the lighting duration of the LED stroboscope (5 microseconds) was not varied. In order to 
avoid further influences from environmental light, a box was constructed. The liquid used did not play any role in this. The entire measurement uncertainty of the actual configuration, adhering to the conditions named and set for the measurements, was therefore estimated at approximately $10 \%-15 \%$.

\section{Summary and Outlook}

The methods presented were largely based on the development of specific algorithms which used the existing hardware and extended its functional scope. This process can be taken into consideration in both the design of the dosing robots and integrated into the existing systems. Largely, these improvements covered the automation of measuring the drop volume.

Thanks to the modular structure of these algorithms, they can be integrated into different dosing systems of similar design and function with just a few specific adjustments. In specific cases, in which there is a need to enhance the values achieved, the suggested alternatives could be applied. There is particular potential in the area of volume measurement with regard to established processes from particle measurement technology.

Future work will include the following topics.

(i) Measuring the drop volume: use of high-speed cameras.

(ii) Evaluation of drop formation: use of high-speed cameras.

(iii) Determining the offset: integration of an additional camera for detecting the three-dimensional trajectory.

(iv) Automatic finding of the dosing parameters.

Software was developed as the basis for the implementation and testing of all processes drawn up. The basic components were used for communication and to control each component in the dosing system. It was possible to integrate the individual algorithms into the program sequence and assign parameters to them via a graphical user interface. In order to be able to port these algorithms into the actual doser software subsequently, the respective algorithms were designed as linked modules.

During the programming, the libraries of the respective manufacturers were used for the hardware access. There was a DLL for the frame grabber and ActiveX control for the components of the dosing system. The operations and subfunctions of the image processing algorithms originated from the OpenCV library. The graphical user interface was based on the Windows API. The program linking these components together was written in Ada 2005. Further measured values of all of the processes investigated could be displayed using this software [23].

\section{References}

[1] A. V. Lemmo, D. J. Rose, and T. C. Tisone, "Inkjet dispensing technology: applications in drug discovery," Current Opinion in Biotechnology, vol. 9, no. 6, pp. 615-617, 1998.
[2] J. W. Hong and S. R. Quake, "Integrated nanoliter systems," Nature Biotechnology, vol. 21, no. 10, pp. 1179-1183, 2003.

[3] A. W. Chow, "Lab-on-a-chip: opportunities for chemical engineering," AIChE Journal, vol. 48, no. 8, pp. 1590-1595, 2002.

[4] P. Calvert, "Inkjet printing for materials and devices," Chemistry of Materials, vol. 13, no. 10, pp. 3299-3305, 2001.

[5] D. J. Hayes, W. R. Cox, and M. E. Grove, "Low-cost display assembly and interconnect using ink-jet printing technology," Journal of the Society for Information Display, vol. 9, no. 1, pp. 9-13, 2001.

[6] Brochure, “Datasheet: BP 211D," Sartorius AG, 2008.

[7] J. Böcker, Chromatographie: Instrumentelle Analytik mit Chromatographie und Kapillarelektrophorese, Vogel, Würzburg, Germany, 1997.

[8] M. Englmann, A. Fekete, I. Gebefügi, and P. Schmitt-Kopplin, "The dosage of small volumes for chromatographic quantifications using a drop-on-demand dispenser system," Analytical and Bioanalytical Chemistry, vol. 388, no. 5-6, pp. 1109-1116, 2007.

[9] P. B. Taylor, S. Ashman, S. M. Baddeley, et al., "A standard operating procedure for assessing liquid handler performance in high-throughput screening," Journal of Biomolecular Screening, vol. 7, no. 6, pp. 554-569, 2002.

[10] Y. Aizu and T. Asakura, "Principles and development of spatial filtering velocimetry," Applied Physics B, vol. 43, no. 4, pp. 209224, 1987.

[11] S. Bergeler and H. Krambeer, "Novel optical spatial filtering methods based on two-dimensional photodetector arrays," Measurement Science and Technology, vol. 15, no. 7, pp. 13091315, 2004.

[12] K. C. Michel, O. F. Fiedler, A. Richter, K. Christofori, and S. Bergeler, "A novel spatial filtering velocimeter based on a photodetector array," IEEE Transactions on Instrumentation and Measurement, vol. 47, no. 1, pp. 299-303, 1998.

[13] J. M. Meacham, M. J. Varady, F. L. Degertekin, and A. G. Fedorov, "Droplet formation and ejection from a micromachined ultrasonic droplet generator: visualization and scaling," Physics of Fluids, vol. 17, no. 10, Article ID 100605, 8 pages, 2005.

[14] A.-S. Yang, J.-C. Yang, and M.-C. Hong, "Droplet ejection study of a Picojet printhead," Journal of Micromechanics and Microengineering, vol. 16, no. 1, pp. 180-188, 2006.

[15] H. Dong, W. W. Carr, and J. F. Morris, "Visualization of dropon-demand inkjet: drop formation and deposition," Review of Scientific Instruments, vol. 77, no. 8, Article ID 085101, 8 pages, 2006.

[16] G. Schröder, Technische Optik, Vogel, Würzburg, Germany, 2002.

[17] G. J. Awcock and R. Thomas, Applied Image Processing, McGraw-Hill, Hightstown, NJ, USA, 1995.

[18] N. R. Pal and S. K. Pal, "A review on image segmentation techniques," Pattern Recognition, vol. 26, no. 9, pp. 1277-1294, 1993.

[19] I. D. Svlabe, "The geometry of basis sets for morphologic closing," IEEE Transactions on Pattern Analysis and Machine Intelligence, vol. 13, no. 12, pp. 1214-1224, 1991.

[20] P. Soille, Morphological Image Analysis: Principles and Applications, Springer, New York, NY, USA, 2003.

[21] Brochure, “Datasheet: XC-ES 50,” Sony Corporation, 2007. 
[22] H. W. Lilliefors, "On the Kolmogorov-Smirnov test for normality with mean and variance unknown," Journal of the American Statistical Association, vol. 62, no. 318, pp. 399-402, 1967.

[23] G. Bradski, “The OpenCV library," Dr. Dobb's Journal, vol. 25, no. 11, pp. 120-125, 2000. 


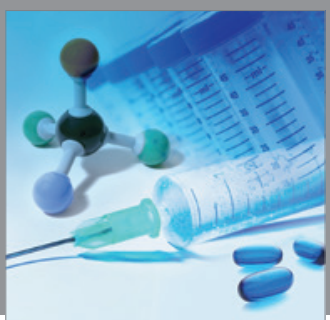

International Journal of

Medicinal Chemistry

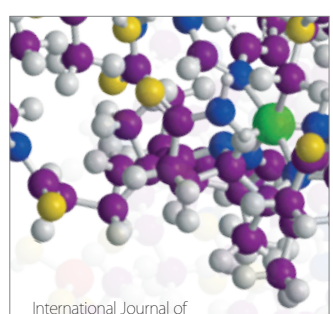

Carbohydrate Chemistry

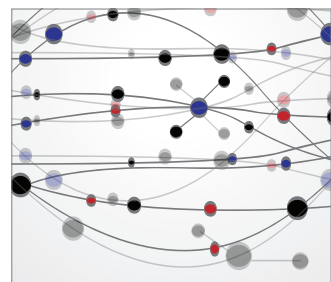

The Scientific World Journal
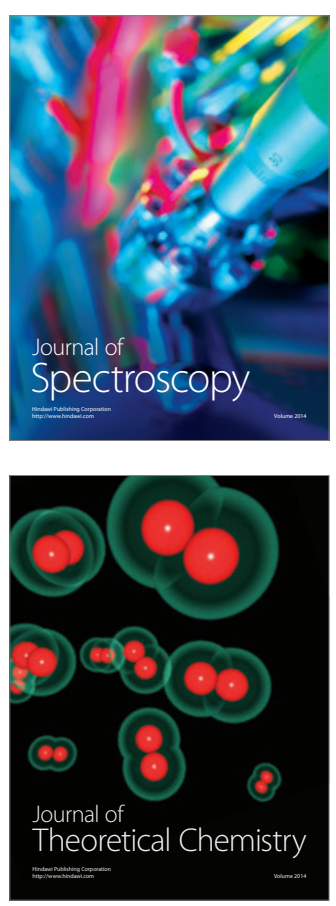
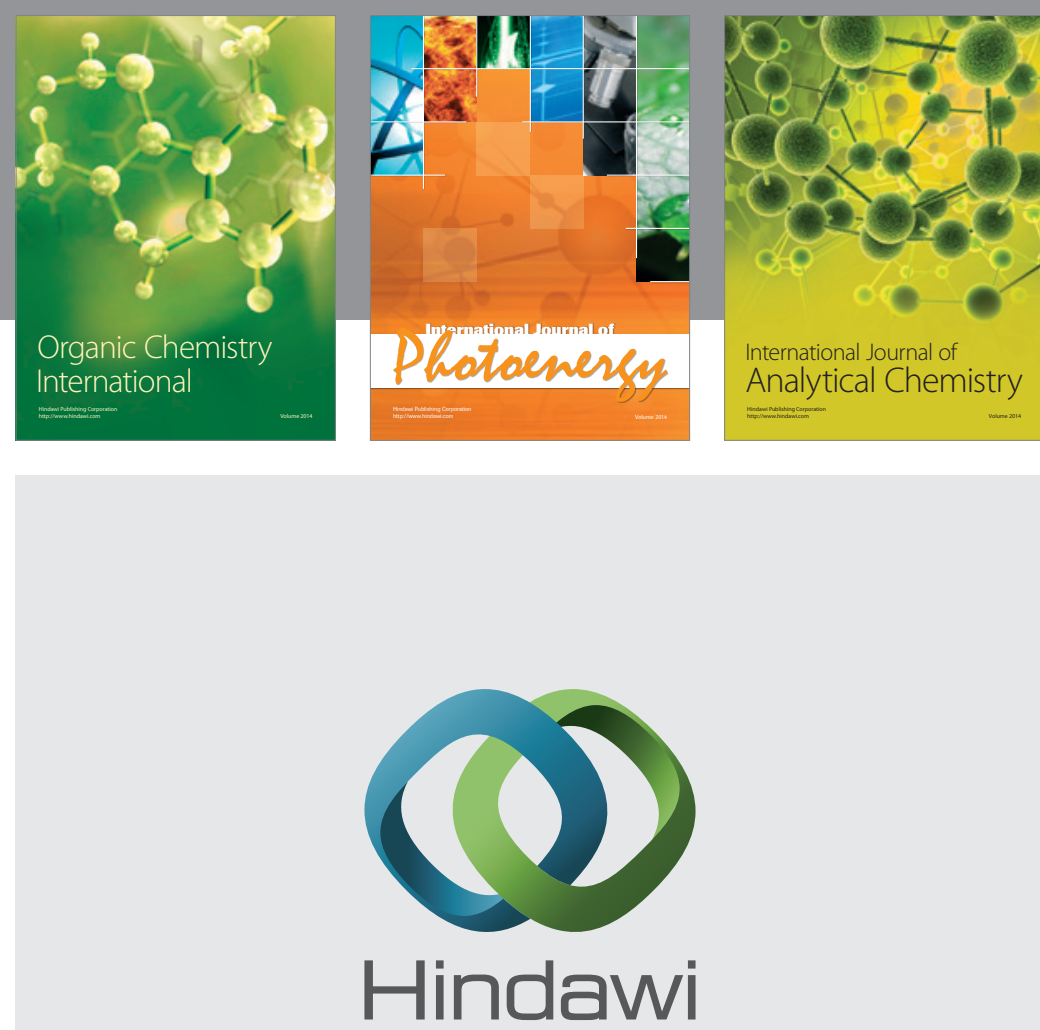

Submit your manuscripts at

http://www.hindawi.com
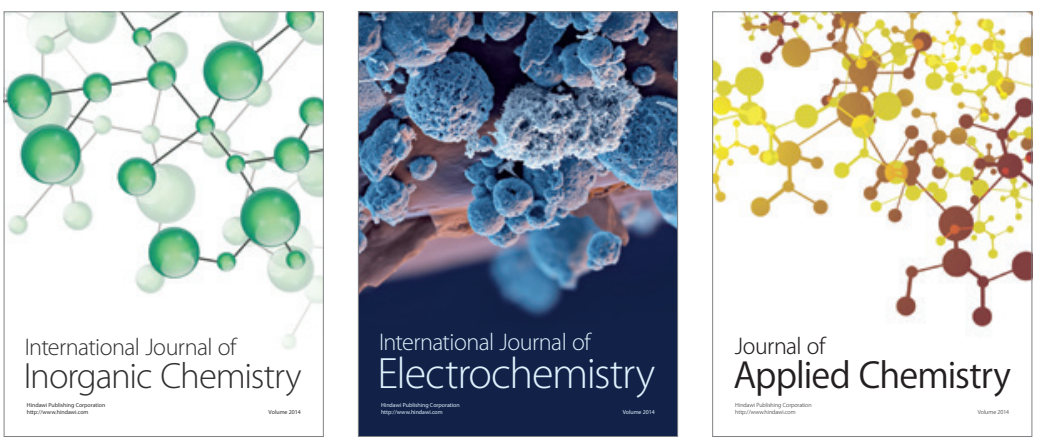

Journal of

Applied Chemistry
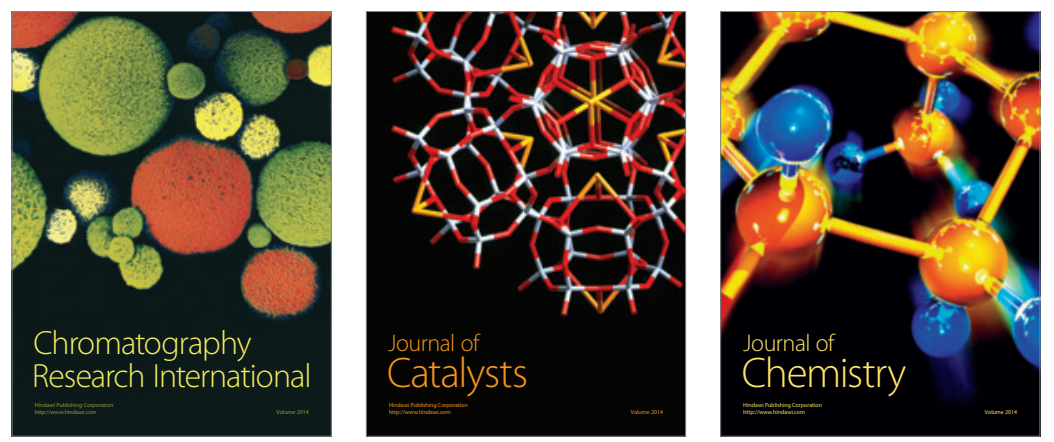
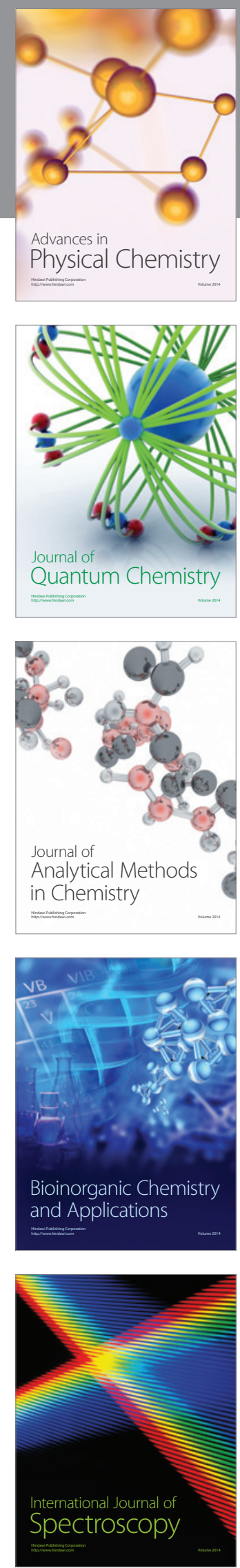